\title{
MINIMIZAÇÃO DA FLEXIBILIDADE EM ESTRUTURAS 3D UTILIZANDO MOT
}

\section{Karilany Dantas Coutinho}

Universidade Federal do Rio Grande do Norte, Departamento de Engenharia

Biomédica, Campus Universitário, Lagoa Nova, Natal, RN, Brasil. CEP: 59072-970. karilany@ufrnet.br

João Carlos Arantes Costa Júnior

Universidade Federal do Rio Grande do Norte, Departamento de Engenharia Mecânica, Campus Universitário, Lagoa Nova, Natal, RN, Brasil. CEP: 59072-970, Fax: (84) 3215-3768, Fone: (84) 32153740, r-207. arantes@ufnet.br

\section{Marcelo Krajnc Alves}

Universidade Federal de Santa Catarina, Departamento de Engenharia Mecânica, C.P. 476, CEP 88010-970, Florianópolis, SC, Brasil. Fax: (48) 2343131, Fone: (48)

3319899.krajnc@emc.ufsc.br

\section{Custódio Leopoldino Brito Guerra Neto}

Universidade Federal do Rio Grande do Norte, Departamento de Engenharia

Biomédica, Campus Universitário, Lagoa Nova, Natal, RN, Brasil. CEP: 59072-970. custodioguerra@yahoo.com.br

\section{Caroline Dantas Vilar Wanderley}

Universidade Federal do Rio Grande do Norte, Departamento de Engenharia

Biomédica, Campus Universitário, Lagoa Nova, Natal, RN, Brasil. CEP: 59072-970. carolinevilar@gmail.com

\section{RESUMO}

Neste trabalho, o Método de Otimização Topológica (MOT) é utilizado para definir a ótima distribuição de massa num pré-definido domínio de projeto, de modo a minimizar a flexibilidade e atendendo a um limite de volume material. Com o objetivo de obter um processo metodológico que seja competitivo e capaz de proporcionar leiautes de alta definição é utilizado um elemento finito tetraédrico de quatro nós, que interpola o campo de densidades relativas além das componentes do campo de deslocamentos. O problema de travamento volumétrico é evitado com a utilização de um esquema de integração seletiva. Para a descrição das propriedades do material, utiliza-se uma formulação microestrutural intermediária artificial denominada SIMP. A formulação possui restrições de estabilidade, que são aplicadas às variáveis de projeto para evitar os problemas de tabuleiro. O problema de ótimo é resolvido pela solução de uma sequiência de problemas de programação não linear sujeito a restrições laterais. Os resultados proporcionaram: um leiaute estrutural bem definido, com nítida distribuição do material e definição do contorno com qualidade proporcional ao tamanho médio do elemento da malha em questão, assim como uma considerável redução no total de variáveis de projeto, devido à utilização do elemento finito utilizado. A formulação mostrou-se promissora de modo a ser utilizada e ampliada para a utilização de processos adaptativos.

\section{PALAVRAS-CHAVE:}

topologia, leiaute, otimização, flexibilidade, MEF. 


\title{
MINIMUM COMPLIANCE OF 3D STRUCTURES WITH TOM
}

\begin{abstract}
The objective of this work is to propose a sharpness and competitive procedure for the determination of the optimum topology of 3D structures. The basic idea behind the concept of Topology Optimization Method (TOM) is the characterization of the layout of the body domain. The finite element refinement procedure is implemented by using a classical tetrahedron finite element with four nodes, with a selective integration scheme to avoid volumetric locking, which interpolates both the displacement and the relative density fields. The checkerboard instability problem is circumvented, by applying the local slope constrained method. In order to obtain the optimum topology we make use of the SIMP microstructure. The formulation of the optimization problem consists in the minimization of the compliance of the structure, subjected to a volume, side and slope constraints. The design variables are defined as the nodal relative densities of the material, defined at each node of the finite element mesh.
\end{abstract}

KEYWORDS:

topology, layout, optimization, compliance, FEM.

\section{MINIMIZAÇÃO DA FLEXIBILIDADE EM ESTRUTURAS 3D UTILIZANDO MOT}

\section{INTRODUÇÃO}

Com o objetivo de definir a topologia ótima de estruturas, Bendsoe \& Kikuchi (1988) proporam o Método de Otimização Topológica (MOT), baseado na Teoria da Homogeneização. Neste método, o problema de otimização topológica é transformado em um problema de redistribuição material sob um pré-estabelecido domínio de projeto. As propriedades efetivas do material composto são estimadas pela Teoria da Homogeneização. O conceito deste método foi utilizado para resolver problemas de minimização da energia interna de deformação por Suzuki \& Kikuchi (1991), Díaz \& Bendsoe (1992), Tenek \& Hagiwara (1993), Bendsoe et. al. (1995) and Krog \& Olhoff (1999). Na literatura, este critério de ótimo também pode ser denominado de minimização da flexibilidade, ou ainda, de modo equivalente, critério de maximização da rigidez. Formulações simplificadas foram propostas por Mlejnek \& Schirrmacher (1993), Yang \& Chuang (1994) e Costa Jr. \& Alves (2000, 2002). Nos trabalhos de Costa Jr. \& Alves $(2000,2002)$ a densidade do material é considerada constante dentro do elemento. Além disso, as propriedades efetivas do material são determinadas por uma equação constitutiva homogeneizada, que depende apenas da densidade relativa do material e é baseada no modelo proposto por Gea (1996).

Neste trabalho será desenvolvido um processo computacional que seja competitivo e eficiente na definição da topologia de estruturas 3D. O MOT a ser desenvolvido, deve ser aplicado ao critério de minimização da energia interna de deformação, sendo as restrições associadas ao problema: a restrição de volume e as restrições laterais sob as variáveis de projeto. Para estabilizar a solução e assim evitar problemas de tabuleiro, impõem-se restrições de estabilidade, de modo a controlar as variações de densidade em cada direção. As equações de estado são aproximadas pelo Método dos Elementos Finitos de Galerkin, com a utilização de elementos tetraédricos de quatro nós que 
interpola o campo de densidades relativas além das componentes do campo de deslocamentos. O modelo material é caracterizado por uma microestrutura artificial do tipo SIMP.

\section{DEFINIÇÃO DO PROBLEMA}

A Fig. 1 caracteriza um processo de otimização de topologia. Aqui, $\Omega$ é o domínio do corpo a ser considerado, o contorno é denotado por $\partial \Omega=\Gamma_{\mathrm{u}} \cup \Gamma_{\mathrm{t}}$ e $\Gamma_{\mathrm{u}} \cap \Gamma_{\mathrm{t}}=\varnothing$, onde $\Gamma_{\mathbf{u}}$ é a parte do contorno com deslocamento prescrito, i.e., $\mathbf{u}=\overline{\mathbf{u}}$ e $\Gamma_{\mathrm{t}}$ é a parte do contorno com tração prescrita, i.e., $\sigma \mathbf{n}=\overline{\mathbf{t}}$. Denota-se $\mathbf{b}$ como a força de corpo prescrita definida em $\Omega \quad$ e $\quad H_{\mathrm{o}}(\Omega)=\mathbf{v} \mid \mathbf{v} \in\left[\begin{array}{ll}H^{1} & \Omega\end{array}\right]^{2}, \mathbf{v}=\mathbf{0} \quad$ em $\mathbf{x} \in \Gamma_{\mathbf{u}} \quad$ e $H=\overline{\mathbf{u}}+H_{\mathrm{o}} \quad$ são os conjuntos das variações e deslocamentos admissíveis respectivamente.

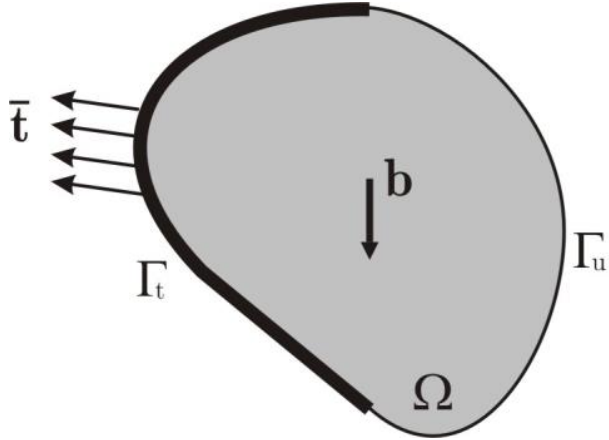

(a) domínio inicial

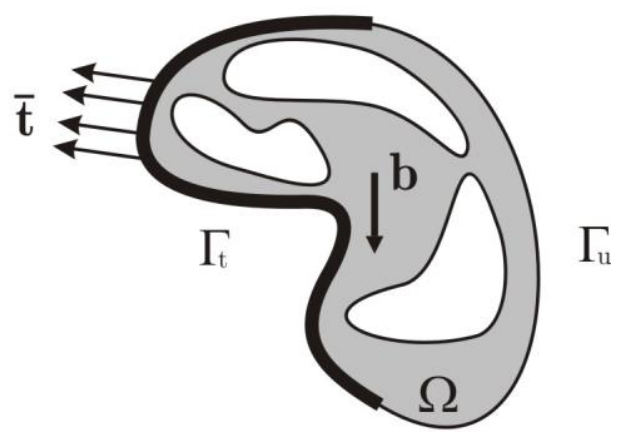

(b) domínio final

Figura 1- Caracterização do problema de otimização de leiaute.

\section{Formulação do Problema}

O problema de otimização tem como objetivo minimizar a flexibilidade de estruturas tridimensionais sob uma restrição de volume e restrições laterais.

Desta forma, o problema resume-se a:

Determinar a distribuição de densidade relativa $\rho$ do material em um dado volume $\Omega$, tal que seja a solução de:

$$
\min _{\rho} l \mathbf{u}=\min _{\rho} \int_{\Omega} \overline{\mathbf{b}} \cdot \mathbf{u} d \Omega+\int_{\Gamma_{t}} \overline{\mathbf{t}} \cdot \mathbf{u} d \Gamma,
$$

sujeito as seguintes restrições:

(i) Restrições de Volume:

$$
\int_{\Omega} \rho d \Omega=V_{o},
$$

(ii) Restrições Laterais

$$
\rho_{\text {inf }}-\rho \mathbf{x} \leq 0 \quad \text { e } \quad \rho \mathbf{x}-\rho_{\text {sup }} \leq 0, \quad \forall \mathbf{x} \in \Omega
$$


(iii) Restrições de Estabilidade

$\left(\frac{\partial \rho \mathbf{x}}{\partial j}\right)^{2}-c_{j}{ }^{2} \leq 0, \quad p / j=x, y$ e $z ; \quad \forall \mathbf{x} \in \Omega$

As constantes $c_{x}, c_{y}$ e $c_{z}$ impõem um limite superior às componentes do gradiente da densidade relativa, Petersson \& Sigmund (1998) e Sigmund \& Petersson (1998).

O campo de deslocamento $\mathbf{u}(\rho(\mathbf{x}), \mathbf{x})$ é solução de:

$a \mathbf{u}, \mathbf{v}=l \mathbf{v}, \quad \forall \mathbf{v} \in H_{\mathrm{o}}$

$$
\therefore \operatorname{div} \sigma+\overline{\mathbf{b}}=0
$$

Onde $\boldsymbol{\sigma}=\mathbf{D}^{H} \rho \boldsymbol{\varepsilon} \mathbf{u}$ e $\overline{\mathbf{u}}=\mathbf{0}$ em $\Gamma_{u}, \boldsymbol{\sigma n}=\overline{\mathbf{t}}$ em $\Gamma_{t}$,

sendo:

$$
a \mathbf{u}, \mathbf{v}=\int_{\Omega} \mathbf{D}^{H} \rho \boldsymbol{\varepsilon} \mathbf{u} \cdot \boldsymbol{\varepsilon} \mathbf{v} d \Omega
$$

Sendo a integral definida na equação (6) aproximada por uma integração seletiva, de modo que:

$$
a(\mathbf{u}, \mathbf{v})=a_{v o l}(\mathbf{u}, \mathbf{v})+a_{\text {dist }}(\mathbf{u}, \mathbf{v})
$$

onde, $a_{\text {vol }}(\mathbf{u}, \mathbf{v})$ são os termos volumétricos e $a_{\text {dist }}(\mathbf{u}, \mathrm{v})$ são os termos de distorção.

$\mathrm{E}$

$$
l \mathbf{v}=\int_{\Omega} \mathbf{b} \cdot \mathbf{v} d \Omega+\int_{\Gamma_{\mathbf{t}}} \mathbf{t} \cdot \mathbf{v} d \Gamma .
$$

\section{Definição do Modelo Material}

Para formular o problema de otimização de leiaute, aplica-se uma técnica de relaxação do problema que consiste na utilização de um material composto caracterizado pela presença de microestruturas porosas, parametrizadas por sua densidade relativa. O modelo microestrutural utilizado é denominado SIMP (Solid Isotropic Material with Penalty), ver Bendsoe (1995) e Bendsoe \& Sigmund (1999), que caracteriza o comportamento constitutivo de um material artificial, e é definido por uma função paramétrica associada com a densidade do material. O modelo material SIMP considera o módulo de Young efetivo, para uma densidade relativa intermediária, $E \rho$, sendo dado por:

$$
E \rho=\rho^{\eta} E_{\mathrm{o}} \text {. }
$$

Aqui, $E_{\mathrm{o}}$ representa o módulo de Young do material sólido e $\eta$ denota o parâmetro de penalização.

A equação constitutiva homogeneizada pode ser definida como:

$$
\boldsymbol{\sigma}=\left[\begin{array}{ll}
\mathbf{D}^{H} & \rho
\end{array}\right] \boldsymbol{\varepsilon}
$$

no qual $\sigma^{\mathrm{T}}=\sigma_{x x}, \sigma_{y y}, \sigma_{z z}, \sigma_{x y}, \sigma_{y z}, \sigma_{z x}$. 
Aplicando o esquema de integração seletiva, teremos que:

$$
\left[\mathbf{D}^{H}(\rho)\right]=\left[\mathbf{D}_{v o l}^{H}\right]+\left[\mathbf{D}_{\text {dist }}^{H}\right] .
$$

Sub-integrando os termos volumétricos:

$$
\left[\mathbf{D}_{v o l}^{H}\right]=\left[\begin{array}{cc}
\mathbf{D}_{11}^{H}(\rho) & 0 \\
0 & 0
\end{array}\right], \varepsilon^{\mathrm{T}}=\left\{\varepsilon_{x x}, \varepsilon_{y y}, \varepsilon_{z z}, 0,0,0\right\}
$$

e integrando os termos de distorção,

$$
\left[\mathbf{D}_{\text {dist }}^{H}\right]=\left[\begin{array}{cc}
0 & 0 \\
0 & \mathbf{D}_{22}^{H}(\rho)
\end{array}\right], \quad \varepsilon^{\mathrm{T}}=\left\{0,0,0, \gamma_{x y}, \gamma_{y z}, \gamma_{z x}\right\}
$$

com



$$
\begin{aligned}
& {\left[\mathbf{D}_{22}^{H} \rho\right]=\frac{E}{21+v}\left[\begin{array}{lll}
1 & 0 & 0 \\
0 & 1 & 0 \\
0 & 0 & 1
\end{array}\right]}
\end{aligned}
$$

sendo $v$ o coeficiente de Poisson.

\section{APROXIMAÇÃO DO PROBLEMA}

\section{Formulação Discreta do Problema}

O problema estrutural é solucionado aplicando o Método do Lagrangeano Aumentado que consiste na solução de uma seqüência de problemas de mínimo com restrições laterais. O elemento finito utilizado na aproximação é o tetraedro de quatro nós, que interpola o campo de densidades relativas além das componentes do campo de deslocamentos.

O problema discreto de otimização de leiaute pode ser formulado como: Determinar $\rho \in \mathbb{R}^{n}$, que seja solução de:

$$
\min _{\rho} f \mathbf{u} \rho,
$$

sujeito as seguintes restrições:

$$
\begin{aligned}
& \int_{\Omega} \rho d \Omega-V_{o} \leq 0 \\
& \rho_{\text {inf }}-\rho \mathbf{x} \leq 0 \quad \text { e } \quad \rho \mathbf{x}-\rho_{\text {sup }} \leq 0, \quad \forall \mathbf{x} \in \Omega
\end{aligned}
$$




$$
h_{e j} \rho=\frac{\sqrt{\left(\frac{\partial \rho}{\partial j}\right)^{2}}}{c_{j}}-1 \quad, \quad \mathrm{p} / j=x, y \text { e } z
$$

para $e=1, \ldots, n_{e} ; \boldsymbol{\rho} \in \mathbf{X} \operatorname{com} \mathbf{X}=\boldsymbol{\rho} \in \mathbb{R}^{n} \mid \rho_{i}^{\text {inf }} \leq \rho_{i} \leq \rho_{i}^{\text {sup }}, i=1, \ldots, n$.

Aplicando o Método do Lagrangeano Aumentado:

1. Definir valores iniciais: $k=0, \lambda^{k}=0, \mu^{k}=\mathbf{0}$, erro $=1,0, \zeta, \boldsymbol{\omega}^{k}$ e tol.

2. Enquanto erro $>$ tol, efetuar laço:

(i) Solução do problema de minimização com restrições laterais

$$
\min \Pi \rho, \lambda, \mu ; \zeta, \omega, \quad \forall \boldsymbol{\rho} \in \mathbf{X}
$$

onde,

$$
\Pi \boldsymbol{\rho}, \lambda, \boldsymbol{\mu} ; \zeta, \boldsymbol{\omega}=f \boldsymbol{\rho}+\frac{1}{\zeta} \sum_{e=1}^{n_{e}} \Lambda_{e} g_{e}, \zeta \lambda_{e}+\sum_{j=1}^{3}\left[\frac{1}{\omega_{j}} \sum_{e=1}^{n_{e}} \Psi_{e}^{j} h_{e}^{j}, \omega_{j} \mu_{e j}\right],
$$

com

$$
\Lambda_{e} g_{e}, \zeta \lambda_{e}=\left\{\begin{array}{cc}
g_{e} g_{e}+\zeta \lambda_{e} & , \quad \text { se } g_{e} \geq-\frac{\zeta \lambda_{e}}{2} \\
-\left(\frac{\zeta \lambda_{e}}{2}\right)^{2}, & \text { se } g_{e}<-\frac{\zeta \lambda_{e}}{2}
\end{array}\right.
$$

e

$$
\Psi_{e}^{j} h_{e}^{j}, \omega_{j} \mu_{e j}=\left\{\begin{array}{cl}
h_{e}^{j} h_{e}^{j}+\omega_{j} \mu_{e j} \quad, \quad \text { se } h_{e}^{j} \geq-\frac{\omega_{j} \mu_{e j}}{2} \\
-\left(\frac{\omega_{j} \mu_{e j}}{2}\right)^{2} \quad, \quad \text { se } h_{e}^{j}<-\frac{\omega_{j} \mu_{e j}}{2} \quad p / j=1, \ldots, 3 .
\end{array}\right.
$$

(ii) Atualização dos multiplicadores de Lagrange

$$
\lambda_{e}^{k+1}=\max \left\{0, \lambda_{e}^{k}+\frac{2}{\zeta} g_{e} \mathbf{x}^{k}\right\}
$$

e

$$
\mu_{e j}^{k+1}=\max \left\{0, \mu_{e j}^{k}+\frac{2}{\omega_{j}} h_{e} \mathbf{x}^{k}\right\} \quad p / j=1, \ldots, 3 .
$$

(iii) Atualização dos parâmetros de penalidade

$$
\zeta^{k+1}=\left\{\begin{array}{cc}
\gamma_{1} \zeta^{k} \quad, \quad \text { se } \gamma_{1} \zeta^{k}>\zeta^{\text {crit }} \quad, \quad \gamma_{1} \in 0,1 \\
\zeta^{\text {crit }}
\end{array}\right.
$$

$\mathrm{e}$ 


$$
\omega_{j}^{k+1}=\left\{\begin{array}{cc}
\beta_{j} \omega_{j}^{k} \quad, \quad \text { se } \beta_{j} \omega_{j}^{k}>\omega_{j}^{c r i t} \\
\omega_{j}^{c r i t}
\end{array} \quad, \quad \beta_{j} \in 0,1 \quad j=1, \ldots, 3 .\right.
$$

(iv) Determinação do erro

$a=\max _{e}\left|\lambda_{e}^{k+1}-\lambda_{e}\right|, b=\max _{e}\left|\mu_{e 1}^{k+1}-\mu_{e 1}\right|, c=\max _{e}\left|\mu_{e 2}^{k+1}-\mu_{e 2}\right|$ e $d=\max _{e}\left|\mu_{e 3}^{k+1}-\mu_{e 3}\right|$

então, erro $=\max a, b, c, d$.

3. Fim do laço.

Desta forma, o problema pode ser formulado como: Dado $\lambda, \mu_{1}, \mu_{2}, \mu_{3} \in \mathbb{R}^{p} \mathrm{e}$ $\zeta, \omega_{1}, \omega_{2}, \omega_{3} \in \mathbb{R}$. O problema consiste em encontrar um $\boldsymbol{\rho}^{*} \in \mathbb{R}^{n}$ tal que:

$$
\boldsymbol{\rho}^{*}=\arg \min \Pi \boldsymbol{\rho}, \lambda, \boldsymbol{\mu} ; \zeta, \boldsymbol{\omega} \quad, \quad \forall \boldsymbol{\rho} \in \mathbf{X} .
$$

\section{PROBLEMAS}

\section{Problema 01}

Considere o problema ilustrado na Fig. 2, o qual consiste em um bloco cujas dimensões são $a=4 \mathrm{~m}, b=7 \mathrm{~m}$ e $c=5 \mathrm{~m}$. No bloco é submetida uma carga vertical prescrita de $\mathbf{P}=5 \times 10^{6} \mathrm{~N}$. O leiaute ótimo é submetido a uma restrição de volume de $\alpha=0,20$. Para simplificar o problema, analisou-se metade da estrutura com 13389 elementos e 3130 nós.

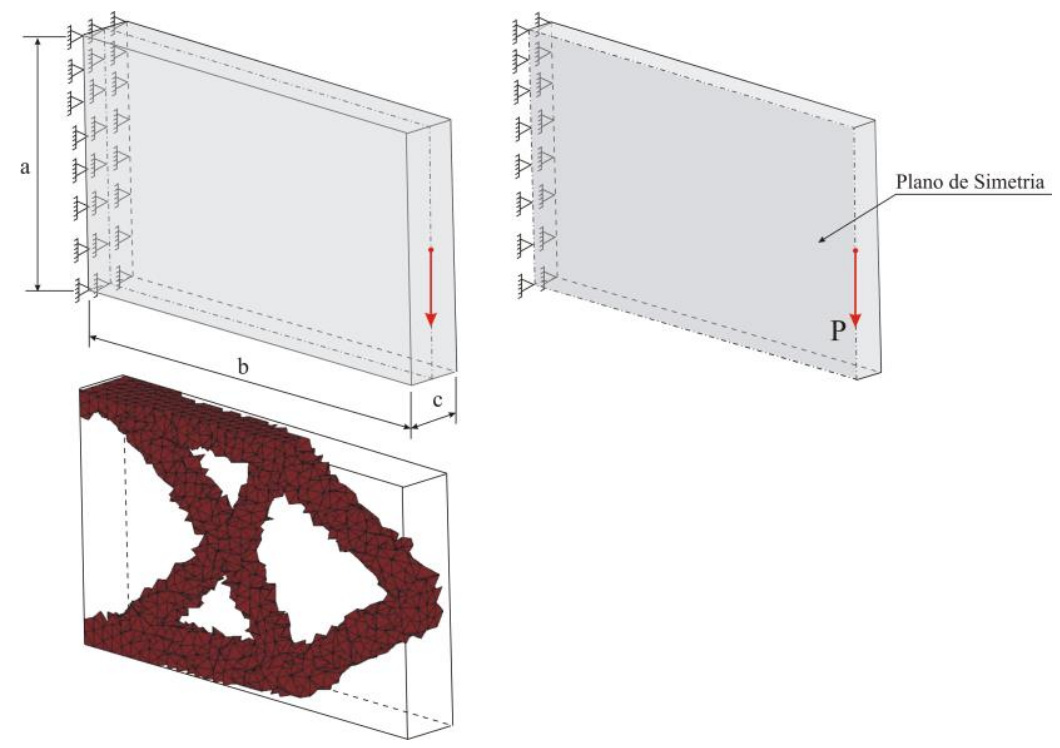

Figura 2- Resultado do Problema 01. 


\section{Problema 02}

Aqui nós consideramos o problema ilustrado na figura na Fig. 3, cujas dimensões são $a=4 m, b=7 m$ e $c=5 \mathrm{~m}$. O bloco está sujeito a uma carga prescrita de $\mathbf{P}=5 \times 10^{6} N$ e restrito a uma fração de volume de 0,20 .

Para simplificar o problema, analisou-se metade da estrutura com 5603 elementos e 1523 nós.
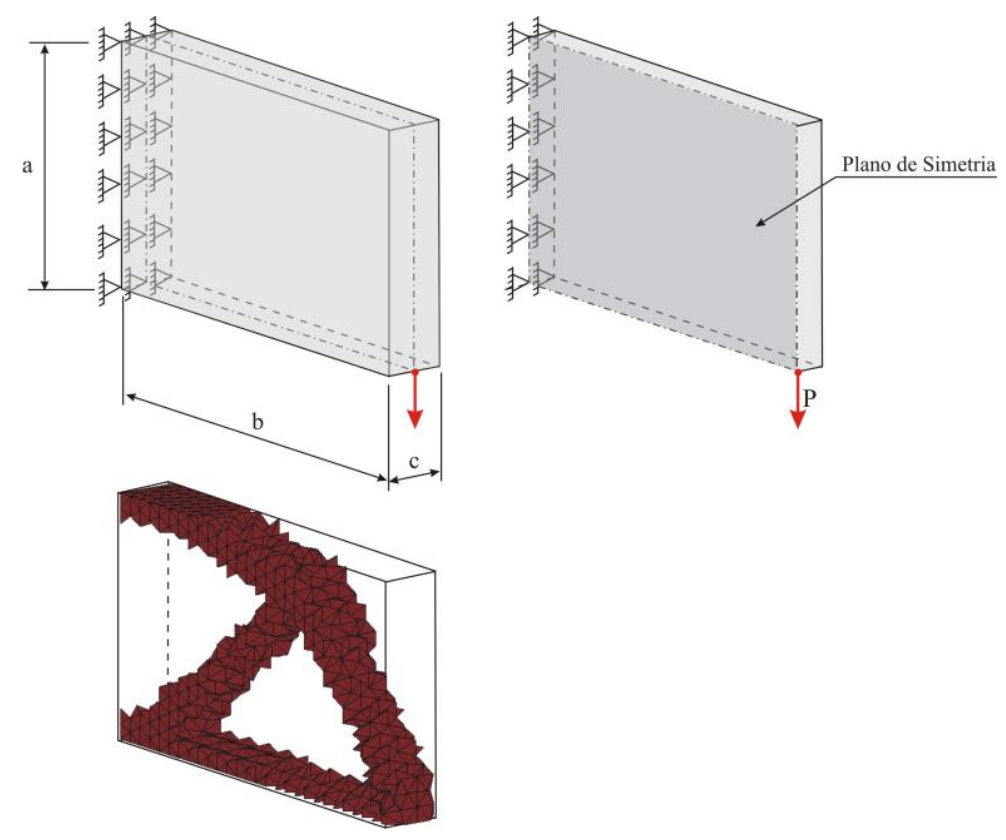

Figura 3- Resultado do Problema 02.

\section{Problema 03}

Neste caso, nós consideramos o exemplo ilustrado na Fig. 4 com dimensões $a=2 m, b=10 m$ e $c=1 m$. O bloco está sujeito a uma carga prescrita de $\mathbf{P}=5 \times 10^{6} N$ e restrito a uma fração de volume de 0,25 . Para simplificar o problema, analisou-se $1 / 4$ de simetria da estrutura com 35555 elementos e 7289 nós.

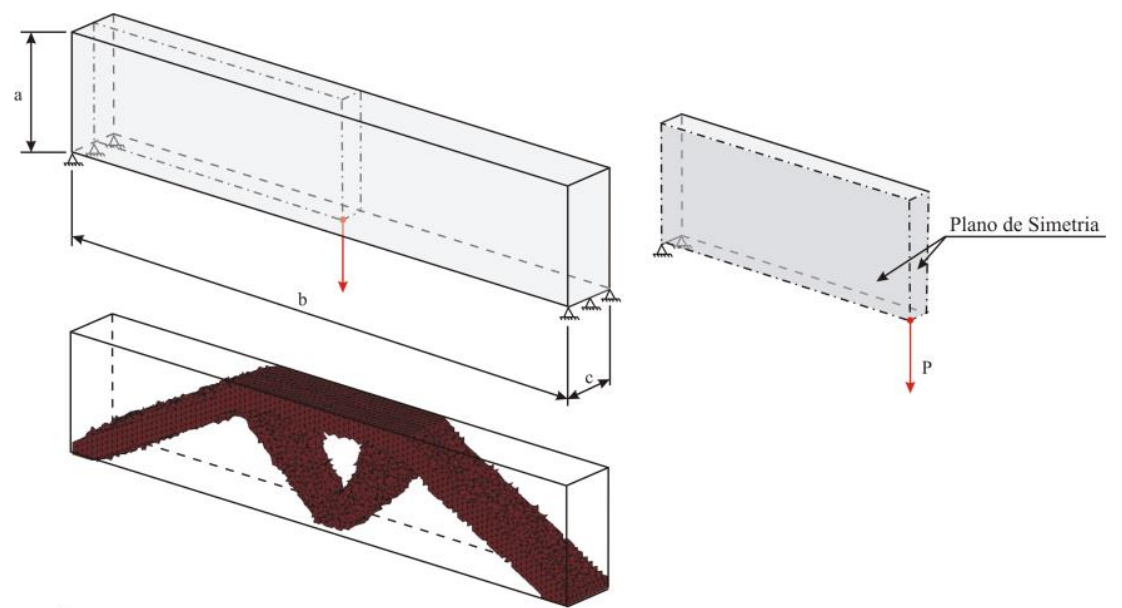

Figura 4- Resultado do Problema 03. 


\section{Problema 04}

Aqui nós consideramos o problema ilustrado na figura na Fig. 5, cujas dimensões são $a=1 \mathrm{~m}, b=1 \mathrm{~m}$ e $c=1 \mathrm{~m}$. O bloco está sujeito a uma carga prescrita de $\mathbf{P}=5 \times 10^{6} N$ e restrito a uma fração de volume de 0,20 . O bloco está fixo em $\bar{\Omega}$ nas três direções $x, y$ e $z$,ou seja, $\bar{\Omega}_{1}=\bar{\Omega}_{2}=\bar{\Omega}_{3}=\bar{\Omega}_{4}$ com deslocamento prescrito 0,0,0 . Para simplificar o problema, analisou-se $1 / 4$ de simetria da estrutura com 16205 elementos e 3218 nós.
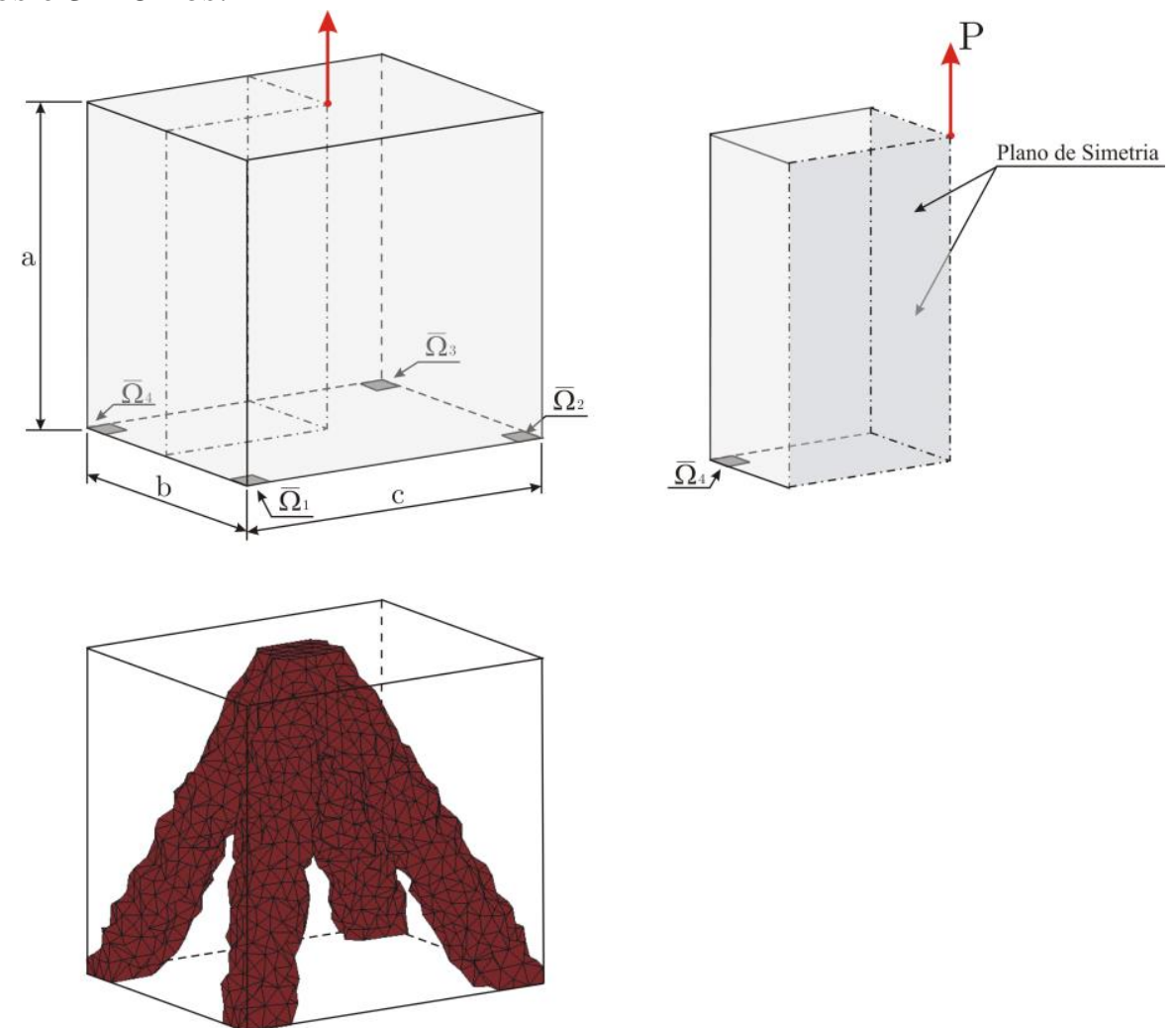

Figura 5- Resultado do Problema 04.

\section{CONCLUSÕES}

De acordo com os resultados obtidos o algoritmo proposto mostrou ser efetivo e robusto na geração de topologias estruturais ótimas para problemas estruturais 3D. A resolução final do contorno material é diretamente dependente do tamanho médio do elemento finito empregado, representando a relação direta com o custo computacional, como pode ser verificado apresenta ótima nitidez com clara disposição de material. Em relação a uma formulação por pixel este método tem a desvantagem do custo computacional para o cálculo da rigidez em cada elemento, mas esta desvantagem pode desaparecer com a implementação de um processo de adaptatividade. A estratégia da implementação de recursos de adaptatividade, ou seja, a implementação de um processo inteligente de refinamento da malha com informações da topologia obtida na malha original, para que seja ainda mais nítido à definição do contorno da topologia com menor custo computacional, está detalhada nos trabalhos de Costa Jr. (2003), Costa Jr. \& Alves (2003a-b). Para a solução do problema discreto de otimização, conforme descrito anteriormente, é aplicado o Método do Lagrangeano Aumentado. Como consequiência, o problema de otimização reduz-se à solução de uma seqüência de problemas de limites laterais do tipo caixa, o qual é resolvido por um método de 
projeção de segunda ordem que usa um método de quasi-Newton sem memória, o solver apresentou muito bom desempenho, numa versão posterior compararemos com a estrutura computacional TANGO de Andreani et al (2004), Andreani et al (2005) e Birgin \& Martinez (2002).

\section{REFERÊNCIAS BIBLIOGRÁFICAS}

1. BENDSOE M. P.; Optimization of Structural Topology, Shape, and Material - , Berlin Heidelberg: Springer-Verlag, 1995, 271 p.

2. BENDSOE M. P., KIKUCHI N.; Generating optimal topologies in structural design using a homogenization method, Comput. Meth. Appl. Mech. Engrg., v. 71(2), p. 197-224, 1988.

3. BENDSOE M. P., SIGMUND O.; Material interpolation schemes in topology optimization, Archive of Applied Mechanics, v. 69, p. 635-54, 1999.

4. CHENG, G. e GUO, X.; E-Relaxed Approach in Structural Topology Optimization, Structural Optimization, vol. 13, pp. 258-266, 1997.

5. COSTA JR. J. C. A.; Otimização Topológica com Refinos H-adaptativos, Tese de Doutorado, UFSC, Santa Catarina, Brasil, 2003.

6. COSTA JR. J. C. A., ALVES M. K.; h-adaptivity procedures of a stress based layout optimization, Cilamce 2003, Ouro Preto-MG, Brasil, CDRom media, 2003 b.

7. COSTA JR. J. C. A., ALVES M. K.; Layout optimization with h-adaptivity of structures, Int. J. Numer. Meth. Engng., v. 58(1), p. 83-102, $2003 \mathrm{a}$.

8. DUYSINX P., SIGMUND O.; New Development in handling stress constraints in optimal material distribution, In 7th AIAA/USAF/NASA/ISSMO Symposium on Multidisciplinary Design Optimization, American Institute of Aeronautics and Astronautics, Saint Louis, Missouri, EUA, paper 98/4906/1-9, 1998.

9. DUYSINX, P. e BENDSOE M. P.; Topology Optimization of Continuum Structures with Local Stress Constraints, Int. J. Numer. Meth. Engng., vol. 43, pp. 1453-1478, 1998.

10. E. G. BIRGIN and J. M. MARTÍNEZ; Large-scale active-set box-constrained optimization method with spectral projected gradients, Computational Optimization and Applications 23, pp. 101-125, 2002.

11. KOHN R. V., STRANG. G.; Optimal design and relaxation of variational problem, Comm. Pure. Appl. Math., 39, p. 1-25, 139-82, 353-77, 1986. 
12. PETERSSON J.; Sigmund O. Slope constrained topology optimization, Int. J. Numer. Meth. Engng., v. 41(8), 1417-34, 1998.

13. R. ANDREANI, E. G. BIRGIN, J. M. MARTÍNEZ and M. L. SCHUVERDT; Augmented Lagrangian methods under the Constant Positive Linear Dependence constraint qualification, Mathematical Programming, 2004.

14. R. ANDREANI, E. G. BIRGIN, J. M. MARTÍNEZ and M. L. SCHUVERDT; On Augmented Lagrangian methods with general lower-level constraints, Technical Report MCDO-050304, Department of Applied Mathematics, UNICAMP, Brazil, 2005.

15. SIGMUND O., PETERSSON J.; Numerical instabilities in topology optimization: a survey on procedures dealing with checkerboards, mesh dependencies and local minima, Structural Optimization, v. 16, p. 68-75, 1998. 